\title{
Alcohol Induced Diabetic Ketoacidosis Exacerbated by an Acute Respiratory Infection with Klebsiella pneumoniae
}

\author{
CALEB DISTEL, STEPHANIE JACOBSON, PATRICIA M. TILLE
}

\begin{abstract}
Ketoacidosis is a metabolic condition that occurs as a result of an insufficient amount of insulin. The lack of insulin results in an increased release of glucose from the liver and an excess of ketone bodies as a result of the breakdown of adipose tissue. This occurs when carbohydrates are unable to be properly processed for needed energy requirements during cellular metabolism. Ketoacidosis is commonly linked to diabetes mellitus. Diabetes mellitus is a condition where the body is unable to produce the proper amount of insulin or is unable to effectively respond to insulin stimulation. Excessive alcohol use can damage the pancreas, reducing insulin secretion. Other conditions such as pneumonia or urinary tract infections can trigger the release of counter-regulatory hormones that may contribute to the decrease in insulin's activity and secretion. Symptoms of diabetic ketoacidosis often include nausea and vomiting, increased thirst and urine production, hyperglycemia, abdominal pain, shortness of breath, confusion, headache, general weakness, fatigue and increased heart rate. If left untreated, diabetic ketoacidosis can lead to more serious complications including circulatory collapse, decreased blood potassium levels, infection and cerebral edema. The following case study presents a complex condition of ketoacidosis associated with a bacterial infection compounded by the patient's history of alcohol abuse.
\end{abstract}

ABBREVIATIONS: CBC - complete blood count, CMP - complete metabolic panel, IV - intravenous fluid, mg/dL-milligrams per deciliter, $\mathrm{mmol} / \mathrm{L}$ millimoles per liter, mM - milli molar, IU/L international unit per liter, $\mathrm{g} / \mathrm{dL}$ - grams per deciliter, $\mathrm{mmHg}$ - millimeters of mercury, $\mathrm{pCO}_{2}$ - partial pressure of carbon dioxide, CT - computed tomography, ICU - intensive care unit, $\mu \mathrm{g} / \mathrm{dL}$ micrograms per deciliter, ng/mL - nanograms per milliliter, U/L - units per liter, $\mathrm{mOsm} / \mathrm{kg}$ - milliosmoles per kilogram, $\mu \mathrm{mol} / \mathrm{L}$ - micromole per liter, $\mathrm{pKa}$ - acid disassociation constant

INDEX TERMS: Ketoacidosis, Klebsiella pneumonia, diabetes mellitus

Clin Lab Sci 2013;26(2):68

Caleb Distel B.S., Medical Laboratory Science Program ${ }^{I}$ South Dakota State University, Brookings, SD

Stephanie Jacobson M.S. MT(ASCP), Rapid City Regional Hospital Rapid City SD

Patricia Tille Ph.D. MT (ASCP), MLS Program, South Dakota State University, Brookings SD

Address for Correspondence: Patricia Tille Ph.D. MT (ASCP), MLS Program, South Dakota State University, Box 2202, Brookings SD 57007, 605-688-6016, pat.tille@sdstate.edu

\section{PATIENT HISTORY}

A 30 year-old Native American male presented to the Emergency Department at 0200 complaining of nausea and vomiting. The patient has a history of alcohol abuse and reported experiencing intermittent episodes of nausea and vomiting, abdominal pain, weakness and a decrease in appetite during the past few months. Initial examination revealed a dry mouth, polyuria, polydipsia without fever and non-radiating abdominal pain in the mid-epigastric region.

The patient's previous medical history included a wrist surgery and no known drug allergies. He denied tobacco and illicit drug use and reported no alcohol use for the past month. Physical examination indicated no cervical or supraclavicular lymphadenopathy, a soft and non-tender abdomen that is not distended and normal 


\section{CLINICAL PRACTICE}

extremity movement. The patients' temperature was $36^{\circ} \mathrm{C}$, pulse was 75 , respiration was 16 breaths per minute, blood pressure was 109 (systolic) over 67 (diastolic) and pulse oximetry showed 99\% oxygen saturation. The patient's eyes, lungs and heart examinations were normal.

\section{INITIAL LABORATORY ANALYSIS}

Initial testing included a complete blood count (CBC), complete metabolic panel (CMP), urinalysis and an arterial blood gas. Abnormal laboratory results are presented in Table 1.

Table 1. Abnormal test results of initial testing

\begin{tabular}{lccc}
\hline Test & Patient Result & Reference Range & Units \\
Urine glucose & 500 & Negative & $\mathrm{mg} / \mathrm{dL}$ \\
Urine ketone & $\geq 80$ & Negative & $\mathrm{mg} / \mathrm{dL}$ \\
Urine bilirubin & Small & Negative & \\
Ictotest & Negative & Negative & \\
AST & 51 & $0-34$ & $\mathrm{IU} / \mathrm{L}$ \\
Albumin & 2.7 & $3.4-5.0$ & $\mathrm{~g} / \mathrm{dL}$ \\
Blood pCO2 & 30.4 & $35-45$ & $\mathrm{mmHg}$ \\
Carbon Dioxide & 16 & $21-32$ & $\mathrm{mmol} / \mathrm{L}$ \\
Anion Gap & 17 & $3-11$ & $\mathrm{mmol} / \mathrm{L}$ \\
Quantitative Ketone & 5.3 & $0.02-0.27$ & $\mathrm{mM}$ \\
Serum Glucose & 272 & $70-110$ & $\mathrm{mg} / \mathrm{dL}$ \\
ALT & 67 & $0-65$ & $\mathrm{IU} / \mathrm{L}$ \\
Blood pH & 7.34 & $7.35-7.45$ & \\
Bicarbonate & 16 & $23-29$ & $\mathrm{mmol} / \mathrm{L}$ \\
\hline
\end{tabular}

Based on these results, the patient's condition is consistent with ketoacidosis associated with underlying diabetes mellitus. Diabetic ketoacidosis results from insufficient insulin available for the metabolism of glucose. Ketoacidosis specifically is a result of an increase in the amount of ketones in the patient's blood. The absence or reduction in insulin results in the release of free fatty acids from adipose tissue when the body is unable to metabolize carbohydrates for energy. The fatty acids are converted in the liver to ketone bodies (acetoacetate and $\beta$-hydroxybutyrate). Ketones have a low pKa (disassociation constant) that causes an increase in the body's acidity (metabolic acidosis), which can cause extensive damage to organs if left untreated. Diabetes mellitus is a major risk factor contributing to the development of metabolic ketoacidosis. In addition, when a patient presents with a secondary infection, such as pneumonia, the demand for insulin increases as the body requires more energy to mount an effective immune response against the ensuing invader.

A variety of laboratory tests can be utilized to diagnose diabetic ketoacidosis. Arterial blood gases are performed to identify the metabolic state of the patient, and in this case acidosis. A venous blood sample is used for additional testing, including the $\mathrm{CBC}$, metabolic panel to measure kidney function and electrolyte levels that are decreased during a state of dehydration. The urinalysis also provides information to substantiate excess levels of glucose in the urine, kidney damage and dehydration. An oral glucose tolerance test may also be used to diagnose the presence of diabetes. The test determines the patient's ability to respond to ingested exogenous glucose. To perform the test, the patient must have a minimum of 150 grams of carbohydrates three days before the test is scheduled, with a 10 to 16 hour fast prior to the test. ${ }^{1}$ The patient is given a 75 gram dose of glucose orally, then the patient's glucose level is checked every 30 minutes over a period of two hours. If the patient's glucose level rises to above 200 $\mathrm{mg} / \mathrm{dL}$ and remains at that level during the two hours, the patient is diagnosed as having diabetes mellitus.

\section{THREE YEARS LATER}

This patient returns to the hospital three years later, being referred from the same nearby clinic as previously. He has complaints of severe upper abdominal pain and anxiety that has lasted over the past week. He does not mention nausea, vomiting, difficulty breathing, chest pains or seizures. Arterial blood gas testing demonstrates a $\mathrm{pH}$ of 6.95 (7.35-7.45), a $\mathrm{pCO}_{2}$ of $10.6 \mathrm{mmHg}(35-$ $45)$ and bicarbonate of $2 \mathrm{mmol} / \mathrm{L}$ (23-29).

The patient has a history of diabetes, reflux esophagitis and chronic alcohol use, although he has not been drinking for the last seven days. Physical examination shows the patient to be drowsy but rousable with deep painful stimuli and has rapid shallow mouth breathing. His blood pressure is 154 systolic over 94 diastolic, heart rate is around $135-145$ beats per minute, respiratory rate is 45 breaths per minute, oxygen saturation is about $98 \%$ and the patient's temperature is $36.8^{\circ} \mathrm{C}$. He has right hypochondriac region tenderness, with normal pupil reactivity to light, no pedal edema or cyanosis. He is able to move all his extremities and has no external injuries or cranial abnormalities. The patient is tachycardic with no murmurs or gallops heard. 


\section{CLINICAL PRACTICE}

The patient was quickly sent to the ICU (intensive care unit). Bicarbonate, IV (intravenous fluid) fluid and insulin were administered to stabilize him and intubated to compensate the respiratory distress. Physician orders included urine toxicology, CMP, lipid panel, serum osmolality and ketones. A CT (computed tomography) scan was performed to evaluate for gallstones and determine pancreas condition. Follow up lab results are presented in Table 2. Additional test results were within normal ranges. The patient also had a positive drug screen for opiates and cannabinoids. Over the next 6 days, the test values returned to near normal ranges. A chest X-ray revealed a bilateral infiltrate in the patient's lungs. Culture of sputum revealed the presence of Klebsiella pneumoniae, which was successfully treated.

Table 2. Abnormal test results following repeated testing

\begin{tabular}{lccc}
\hline Test & Patient Result & Reference Range & Units \\
WBC Count & 14.7 & $3.7-9.6$ & $\times 10^{3} / \mu \mathrm{L}$ \\
Neutrophils & 59 & $46-70$ & $\%$ \\
Lymphocyte & 11 & $15-47$ & $\%$ \\
Urine Protein & 100 & Negative & $\mathrm{mg} / \mathrm{dL}$ \\
Serum Iron & 20 & $50-175$ & $\mu \mathrm{g} / \mathrm{dL}$ \\
TIBC & 184 & $250-450$ & $\mu \mathrm{g} / \mathrm{dL}$ \\
Ferritin & 1479.3 & $3-244$ & $\mathrm{ng} / \mathrm{mL}$ \\
Calcium & 6.8 & $8.5-10.1$ & $\mathrm{mg} / \mathrm{dL}$ \\
Lipase & 1083 & $20-342$ & $\mathrm{U} / \mathrm{L}$ \\
Bicarbonate & 16 & $23-29$ & $\mathrm{mmol} / \mathrm{L}$ \\
Chloride & 115 & $96-106$ & $\mathrm{mmol} / \mathrm{L}$ \\
CO2 & 4 & $21-32$ & $\mathrm{mmol} / \mathrm{L}$ \\
Anion Gap & 21 & $3-11$ & $\mathrm{mmol} / \mathrm{L}$ \\
Quantitative Ketone & 103.1 & $0.02-0.27$ & $\mathrm{mM}$ \\
Glucose & 308 & $70-110$ & $\mathrm{mg} / \mathrm{dL}$ \\
HgbA1C & 13.4 & $4.8-6.0$ & $\%$ \\
Osmolality & 324 & $275-300$ & $\mathrm{mOsm} / \mathrm{kg}$ \\
Ammonia & $<125$ & $<36$ & $\mu \mathrm{mol} / \mathrm{L}$ \\
Triglyceride & 7391 & $<200$ & $\mathrm{mg} / \mathrm{dL}$ \\
\end{tabular}

\section{DISCUSSION}

The primary condition affecting the patient during his admissions was ketoacidosis. This condition is caused by the breakdown of body fats in order to supply cells with energy when a defect in carbohydrate metabolism occurs, usually due to a loss of insulin or insulin activity. ${ }^{2}$ Diabetes mellitus is commonly associated with the development of ketoacidosis. A decrease in the amount of insulin can be caused by an illness, which can cause the production of counter-regulatory hormones. This causes a reduction in the creation and secretion of insulin, leading to a hyperglycemic state.
Conditions that are most often associated with triggering ketoacidosis include pneumonia and urinary tract infections. ${ }^{3,4}$ Other conditions that can contribute to decreased insulin include stress, surgery, heart attack, stroke and alcohol or drug abuse. The condition usually presents with excessive thirst, increased urination, general weakness or fatigue, nausea and vomiting, abdominal pain, loss of appetite, shortness of breath with an increased respiratory rate. Outward symptoms include a fruity smelling breath, confusion, headache, muscle stiffness, low blood pressure and an increased heart rate. More specific signs include a high blood sugar level and a high urine ketone.

Development of ketoacidosis begins with reduced action of insulin. Decreased production or decreased response to insulin such as that seen in diabetes mellitus causes an increase in glucagon secretion, triggering the liver to begin glycogenolysis or gluconeogenesis to increase blood glucose levels. This creates a hyperglycemic state in the patient since the carbohydrates are not being transferred into cells by insulin. The decreased insulin also causes lipolysis to increase, breaking down adipose tissue in order to supply energy for the cells. ${ }^{1}$ Ketones are formed from fat breakdown, and the accumulation of the ketones creates an acidotic state. Bicarbonate concentration shows a corresponding decrease. Alternative compensation is usually needed to buffer the ketones. This usually involves hyperventilation in order to remove $\mathrm{CO}_{2}$ from the system. The loss of bicarbonate along with the increased ketones creates an elevated anion gap in the patient's blood. ${ }^{1}$ Eventually the glucose and ketones are flushed out through the kidneys along with an excessive amount of water, which increases electrolyte loss including potassium, magnesium and calcium.

Underlying conditions may contribute to ketoacidosis by further decreasing the level of insulin in the patient or by increasing acidic products in the blood. Pancreatitis commonly contributes to ketoacidosis. Pancreatitis involves inflammation of the pancreas, which causes the enzymes to pool and damage the beta Islet of Langerhans cells, reducing the secretion of insulin. ${ }^{5}$ Excessive alcohol use may cause pancreatic inflammation by further destroying the beta pancreatic cells. Metabolism of alcohol impairs hepatic gluconeogenesis by preventing the conversion of lactate to pyruvate. $^{6,7}$ This results in decreased insulin 


\section{CLINICAL PRACTICE}

secretion, increased lipolysis, impaired fatty acid oxidation, ketogenesis and increased counter-regulatory hormones, all of which contribute to the ketoacidotic state.

The infection with $K$. pneumoniae is significant. This infection in the lungs can trigger the development and release of stress hormones, including epinephrine and cortisol, which are counter-regulatory hormones against insulin. As these hormones are increased, insulin secretion is reduced, glucose entry into cells is decreased and gluconeogenesis and glycogenolysis in the liver is increased, creating a hyperglycemic state, fostering conditions that contribute to diabetic ketoacidosis.

Ketoacidosis has a mortality rate in the range of $1 \%$ $10 \%$. Death is normally caused by circulatory collapse, hypokalemia, infection or cerebral edema. ${ }^{8,9}$

Treatment of ketoacidosis involves replacing lost fluid and electrolytes using an IV. Insulin is administered in order to allow glucose to be absorbed into the cells, usually when a hyperglycemic state is above 300 $\mathrm{mg} / \mathrm{dL} .10$ This will also slow down the action of glucagon and lipase, which will help to reduce the amount of ketones in the blood. Bicarbonate can also be administered in order to control the patient's acidotic state. When the cause of ketoacidosis is alcohol related, thiamin and vitamins maybe added to the IV, followed by an infusion of $5 \%$ dextrose with $0.9 \%$ saline. ${ }^{11,12}$

\section{CONCLUSION}

The patient was diagnosed with diabetic ketoacidosis along with new onset diabetes during his first admission. He had a history of alcohol abuse, which contributed to his acidotic state. The patient presented with increased liver function test results that correspond to diabetic ketoacidosis, as well as alcohol damage. During his second admission, he presented with poorly controlled diabetes subsequent to diabetic ketoacidosis. The patient had an increased anion gap and decreased bicarbonate, which caused increased respiration, low $\mathrm{pCO}_{2}$ and tachycardia. The patient's second admission was complicated with acute pancreatitis that developed from continued alcohol use and poorly controlled diabetes. It is demonstrated by an extremely high lipase and abdominal pain. The acute pancreatitis may also be contributing to acute respiratory distress syndrome. The acute respiratory infection with $K$. pneumoniae exacerbated the ketoacidosis but was treated successfully. The patient's drowsiness at admission was due to Ativan, alcohol withdrawal and severe acidosis. After the patient was treated, Gemfibrozil and Levemir insulin were prescribed to keep his triglyceride and insulin levels under control. It was also recommended that he change to a low fat diet and reduce alcohol consumption in order to prevent the reoccurrence of his ketoacidosis. He was also told to return at a later date to monitor his blood sugar and lipid levels.

\section{REFERENCES:}

1. Wilson V. Diagnosis and Treatment of Diabetic Ketoacidosis. Emerg Nurse 2012;20(7);14-9.

2. Wolfsdorf J, Craig ME, Daneman D, Dunger D, Edge J, Lee WR et. Al. Diabetic ketoacidosis. Pediatr Diabetes 2007;8:2843.

3. Gavrielatos G, Ioannidis I, Lionakis N. et. al. Clinical and Laboratory Characteristics of Diabetic Keotacidosis in Adult Diabetic Patients. Internet Journal of Endocrinology 2007;3:3.

4. Umpierrez GE, Kitabchi AE. Diabetic Ketoacidosis: Risk Factors and Management Strategies. Treat Endocrinol 2003;2:95-108.

5. Manikkan AT. Hyperlipasemia in Diabetic Ketoacidosis. Clin Diabetes 2013;31:31-2.

6. Hockenhull J, Dhillo W, Andrews R, Peterson S. Investigation of markers indicate and distinguish death due to Alcoholic Keotacidosis, Diabetic Ketoacidosis and Hyperosmolar Hyperglycemic State using post-mortem samples. Forensic Sci. Int. 2012;214:142-7.

7. Elliott S, Smith C, Cassidy D. The post-mortem relationship between beta-hydroxybutyrate (BHB), acetone and ethanol in ketoacidosis. Forensic Sci. Int. 2010;198:53-7.

8. Ali Z, Levine B, Ripple M, Fowler DR. Diabetic ketoacidosis: a silent death. Office of the Chief Medical Examiner, State of Maryland, Baltimore MD. 2012;33:189-93.

9. Centers for Disease Control and Prevention, Morbidity and Mortality Weekly Report MMWR. 2012;61:869-72.

10. Crump V. Hyperglycemic Crisis. RN. 2004;67:23-7.

11. Wrenn KD, Slovis CM, Minion GE, Rutkowski R. The Syndrome of Alcoholic Ketoacidosis. Am J Med 1991;91:11929.

12. AdroguiA, HJ, Madias NE. Management of Life-Threatening Acid-Base Disorders. N. Engl. J. Med. 1998;338:26-34. 